Check for updates

Cite this: RSC Adv., 2017, 7, 46045

Received 26th May 2017

Accepted 26th August 2017

DOI: $10.1039 / c 7 r a 05912 d$

rsc.li/rsc-advances

\section{pH sensitive mesoporous nanohybrids with charge-reversal properties for anticancer drug delivery}

\author{
Bozhen Wu,,$^{\mathrm{a}}$ Shunshu Deng, $\uparrow^{\mathrm{b}}$ Shihao Zhang, ${ }^{\mathrm{b}}$ Jia Jiang, ${ }^{\star d}$ Baosan Han*c \\ and Yulin $\mathrm{Li}$ (iD *ab
}

The surface/interface state of nanomaterials plays a key role on their biomedical applications. Nanotechnology offers a versatile means to develop nanoparticles with well-defined architecture. In this study, mesoporous silica nanoparticles were firstly loaded with an anticancer drug (doxorubicin, DOX), which were then decorated with a cationic oligomer (low molecular weight polyethyleneimine, LPEI) to acquire an increased surface charge. The resulting particles were further self-assembled with negativecharged bovine serum albumin (BSA) as natural protein nanoblocks to offer surface charge tunability. The resulting mesoporous nanohybrids (MDPB) acquired charge-reversal ability, which presented negative charge under biological conditions (beneficial to biocompatibility), while displaying a positivecharged state under acidic conditions mimicking the tumor extracellular microenvironment (favoring cell uptake or tumor penetration). Furthermore, the nanohybrids not only allowed for an effective loading of DOX drug, but also accelerated its release under acidic tumor microenvironments in a sustainable way. In vitro biological study indicated that the DOX-free nanoparticles were biocompatible, while MDPB exerted good cytotoxicity against cancer cells, suggesting their promise for therapeutic delivery application.

\section{Introduction}

Facing the increasing threat of cancer diseases, how to overcome the multidrug resistance and improve therapeutic bioactivity is still a challenging topic in the field of anticancer research. ${ }^{1-5}$ Even though small anticancer drugs (e.g., doxorubicin (DOX) and paxlitaxel (PTX)) can be encapsulated into some liposomal-based nanoformulations and employed for cancer treatment at a clinical and/or trial clinical level, ${ }^{3-5}$ success has never been achieved because the current nanocarriers have low tumor penetration ability as well as passive release properties. ${ }^{3-5}$ Therefore, it is important to develop a kind of nanocarriers which can carry drug(s) to penetrate through solid tumor and smartly deliver it (them) to kill cancer cells. ${ }^{6}$

${ }^{a}$ College of Materials Science and Engineering, Zhejiang University of Technology, Hangzhou, 310014, China

${ }^{b}$ The State Key Laboratory of Bioreactor Engineering, Key Laboratory for Ultrafine Materials of Ministry of Education, Key Laboratory for Ultrafine Materials of Ministry of Education, Engineering Research Centre for Biomedical Materials of Ministry of Education, East China University of Science and Technology, Shanghai 200237, China. E-mail: yulinli@ecust.edu.cn

'Department of General Surgery, Laboratory of General Surgery, School of Medicine, Xinhua Hospital, Shanghai Jiao Tong University, Kongjiang Road No. 1665, Shanghai 200092, China. E-mail: hanbaosan@126.com

${ }^{d}$ Department of Sports Medicine, Shanghai $6^{\text {th }}$ People's Hospital, Shanghai, 200237, China. E-mail: jessicajj19@hotmail.com

$\dagger$ The authors gave equal contribution to this work.
Normally, negative-charged nanoparticles have better biocompatibility than the positive-charged one. ${ }^{7,8}$ It is also known that tumors assume more acidic extracellular environment ( $\mathrm{pH}$ 6.5) as compared to the normal biological conditions. ${ }^{11,12}$ For nanomedicines used for intravenous injection application, it is better that they present negative charge during blood circulation (to decrease protein adsorption in plasma as well as blood cell trapping), while transformed into positivecharged ones under acidic extracellular environment ( $\mathrm{pH}$ 6.5) to enhance their interactions with tumor cells with a negativecharged membrane. ${ }^{\mathbf{9 , 1 0}}$ Nanoparticles with such pH-sensitivity are called as charge-reversal nanosystems. ${ }^{\mathbf{1 1 2}}$ To obtain this purpose, some $\mathrm{pH}$-sensitive chemical bonds (e.g., $\beta$-carboxylic acid) has been grafted onto some polymers with positive charges to transform them into a temporary negative ones, which can be again triggered into positive state upon their arrival at tumor site to enhance their interactions with cancer cells. ${ }^{13}$ However, this kind of modification is involved in a series of chemical reactions, which greatly complicated the fabrication process and increased the production cost. ${ }^{13}$

Herein, we firstly prepared mesoporous silica nanoparticles (MSN) which have been widely used for therapeutic delivery study because of their biocompatibility, biodegradability and controllable structure. ${ }^{\mathbf{1 4}}$ After that, MSN with mesoporous structure was used to encapsulate doxorubicin (DOX, as a model anticancer drug). For tuning their surface charge, cationic hyperbranched polyethylenimine with low molecular weight 


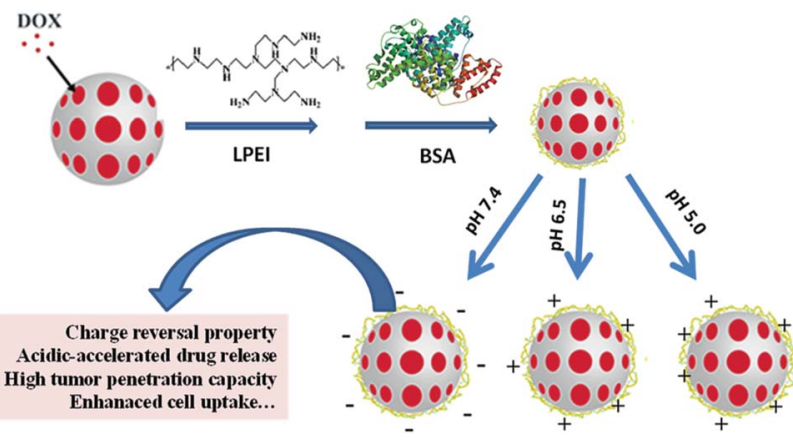

Scheme 1 Schematic representation of how to prepare MDPB nanohybrids via decoration of doxorubicin (DOX)-loaded mesoporous silica nanoparticles (MSN) with low molecular weight PEI (LPEI), followed by introduction with bovine serum albumin (BSA) through a self-assembly approach. Charge-reversal property can be achieved by adjustment of the ratio of cationic LPEI and anionic BSA, which can improve tumor penetration, cancer cell uptake and acidic-accelerated drug delivery targetability, resulting in good cytotoxicity against cancer cells.

(LPEI) which displays less cytotoxicity than high molecular weight PEI has been assembled onto MSN/DOX (MD) to acquire positive charges of LPEI, where its protonation effect may offer $\mathrm{pH}$ sensitivity in drug release. ${ }^{15,16}$ Then, the LPEI-modified MSN nanosystems (MDP) were further decorated with a natural protein (bovine serum albumin, BSA) with negative charges for further adjustment of the nanoparticle surface to enable charge reversal property. As a kind of main component protein existing in the blood plasma, the coverage of BSA on the nanocarriers may protect them from trapping by blood cells. ${ }^{17}$ Furthermore, BSA has a nanosized architecture $(\sim 7 \mathrm{~nm})$, which may act as a temporary nanoblocks to block DOX leakage, while its biodegradability may enable an achievement of a sustainable therapeutic delivery. ${ }^{17,19,20}$ Also, the process for preparation of the mesoporous nanoparticles is very environment-friendly and not involved in any organic solvent (Scheme 1). The results indicated that MDPB nanoparticles effectively encapsulated DOX, which can be released in an acidic-accelerated drug release way under both acidic conditions mimicking extracellular and intracellular microenvironments. Furthermore, the nanonparticles themselves were biocompatible, and delivered DOX to cancer cells to present good cytotoxicity against cancer cells.

\section{Results and discussion}

\section{Synthesis and characterization of charge-reversal mesoporous} nanohybrids

A sol-gel chemistry approach was used to synthesize mesoporous silica nanoparticles (MSN) through an emulsion method. After that, cationic doxorubicin was mixed with MSN to get DOX-loaded samples (MD), which was assembled with PEI with low molecular weight (LPEI) to offer MDP nanoparticles, followed by decoration with BSA to obtain MDPB nanohybrids. LPEI introduction can endow the nanoparticles with positive charges, which can be balanced via assembly with negative BSA to obtain samples with charge-reversal property. The advantage of using LPEI, instead of PEI with high molecular weight, is that the former is more biocompatible than the latter. ${ }^{21,22}$ Through optimization, it was found that decoration of MD with $0.2 \mathrm{mg} \mathrm{mL}{ }^{-1}$ LPEI1 and $0.1 \mathrm{mg} \mathrm{mL}^{-1}$ BSA (this sample was named as MDPB_0.1) can endow the nanoparticles with chargereversal property upon $\mathrm{pH}$ variation from normal conditions (pH 7.4) to acidic tumor extracellular microenvironments (pH 6.5) (Fig. 1).

Generally, BSA decoration did not affect the drug loading capacity of the formed nanohybrids. The encapsulation efficiency of the nanohybrids of all studied BSA was maintained at $\sim 60 \%$ (Fig. 2). For morphological analysis, the samples were imaged via Transmission Electron Microscopy. As can be seen from Fig. 3, all the samples assumed as a sphere shape with diameter around $100 \mathrm{~nm}$. The MD present an obvious mesoporous structure, while surface modification resulted in a blurring on the nanoparticles, especially for MDPB nanohybrids, suggesting a successful coating of LPEI and BSA onto the surface of MSN nanoparticles.

\section{In vitro drug release study}

As mentioned above, it is important to improve the controllability of nanomedicines to increase their therapeutic bioactivity. ${ }^{23-25}$ Even though MSN nanoparticles own good porous

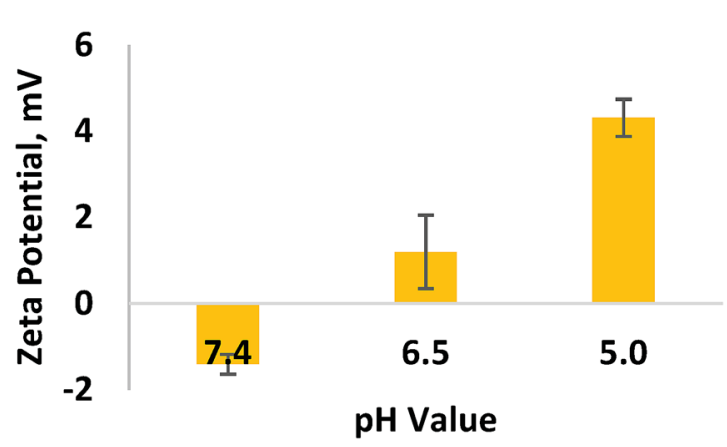

Fig. 1 The $\zeta$-potentials of MDPB_0.1 samples in phosphate buffered saline (PBS) at different $\mathrm{pH}$ values ( $\mathrm{pH} 7.4,6.5$ and 5.0)

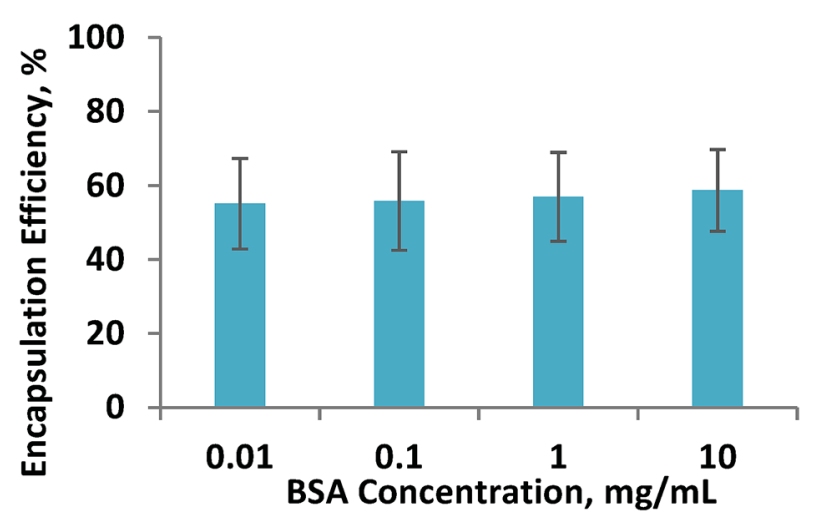

Fig. 2 How the BSA amount which has been introduced on the nanohybrids affects the drug loading capacity of the nanohybrids. 

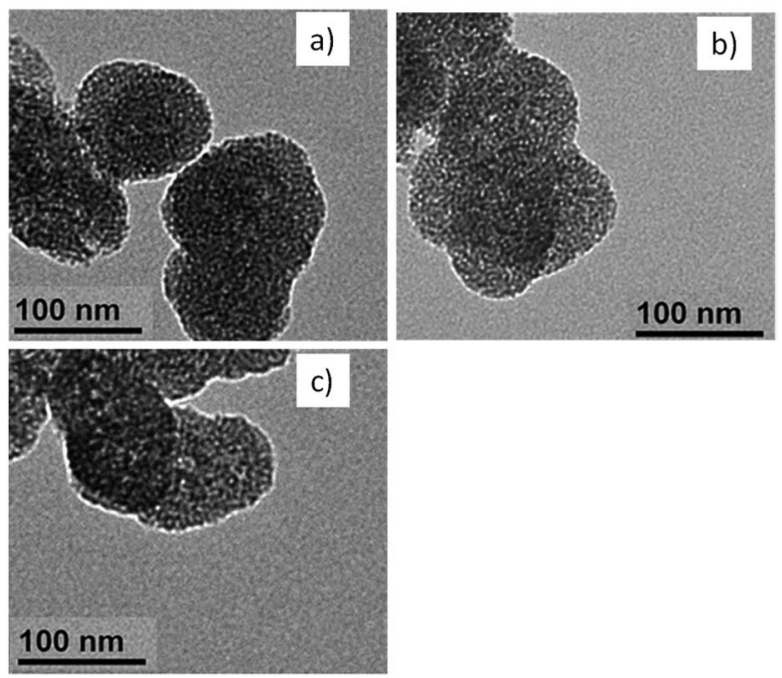

$100 \mathrm{~nm}$

Fig. 3 Morphological properties of (a) MD, (b) MPD and (c) MDPB_0.1 nanohybrids observed by a Transmission Electron Microscope (TEM).

structure, their pore openness is not beneficial to achieving the sustainability in drug release. Our idea in this study is to introduce a temporary protective layer on their surface by assembling a positive oligomer polyethylenimine (LPEI) on its surface, followed by decoration with bovine serum albumin (BSA) which has a nanosized architecture of $\sim 7 \mathrm{~nm} .{ }^{17}$ The existence of BSA in the outer layer may act as temporary nanoblocks to improve the controllability of DOX release from the nanoparticles, thus reducing its burst release.

To verify our hypothesis, the release behaviors of unmodified DOX-loaded MSN (MD), LPEI-coated MD before (MDP) and after BSA (MDPB) treatment were studied under PBS solution at $\mathrm{pH}$ 7.4. As shown in Fig. 4 a, a burst release ( $8 \mathrm{~h}, \sim 65 \%$ ) occurred for MD systems. Although the physical coating of LPEI onto MSN decreased DOX release ability in some degree, the release rate was still quite high ( $8 \mathrm{~h}, 54 \%)$. As comparison, MDPB presented a limited DOX release (43\%) up to $8 \mathrm{~h}$, suggesting the successful BSA coating onto the LPEI layer to sustain the release rate of the encapsulated DOX from the nanoparticles.

Generally, pH responsiveness is a useful bioactive stimulus for achievement of anticancer drug release controllability, since various solid tumors present acidic extracellular microenvironment (pH 6.5) and intracellular compartments (e.g., endo/ lysosomal compartments) display a little bit more acidic state (pH 5.0). ${ }^{26,27}$ In order to check their $\mathrm{pH}$ sensitivity, the DOX release behaviors of the nanoparticles under both physiological $(\mathrm{pH}$ 7.4) and acidic (pH 6.5 and 5.0) conditions were investigated. As can be seen from Fig. $4 \mathrm{~b}$, the decrease of $\mathrm{pH}$ value significantly increased the release capacity of DOX from MDPB. For instance, after $8 \mathrm{~h}$ incubation, their cumulative releases in PBS solution at $\mathrm{pH} 7.4,6.5$ and 5.0 were about $43 \%, 55 \%$ and $63 \%$, respectively. This is important indicator for targetable drug delivery, because during blood circulation period most DOX can be protected to be released from the nanoparticles. However, after their arrival around the tumor site and/or uptake by cancer cells presenting acidic microenvironments, DOX can
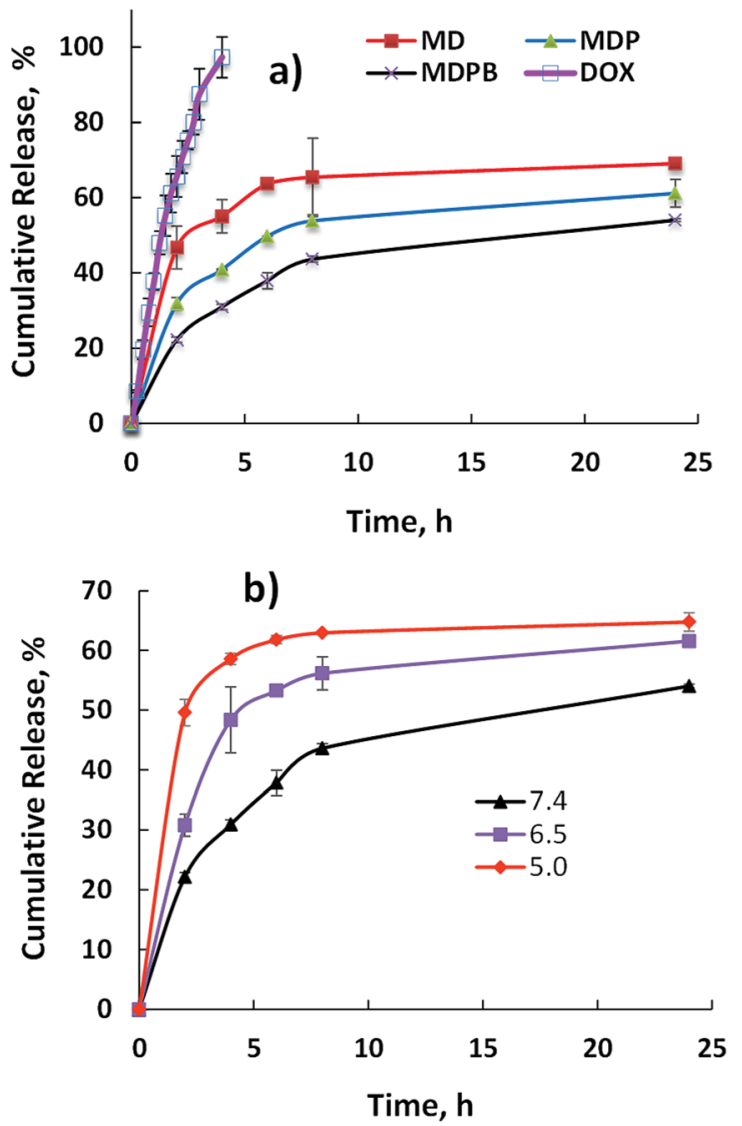

Fig. 4 DOX cumulative release from the nanohybrids in PBS at $37^{\circ} \mathrm{C}$ from (a) free DOX, MD, MDP and MDPB at pH 7.4; (b) MDPB_0.1 at different $\mathrm{pH}$ values $(7.4,6.5$ and 5.0).

be released in an accelerated manner to increase its anticancer bioactivity and decrease its side effects. ${ }^{28-30}$ Since PEI tends to undergo protonation at acidic conditions (low $\mathrm{pH}$ values), which may lead to the PEI shell swelling of the nanoparticles, resulting in an easier diffusion of protonated DOX with higher solubility from them to accelerate its release capacity. The $\mathrm{pH}$ sensitivity of the MDPB carriers may be used to improve their therapeutic targetability to tumors by limiting drug leakage during their blood circulation while allowing for a timely delivery of the drug upon arrival at tumor site and/or uptake by

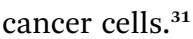

\section{In vitro biological study}

For biomedical applications, the native nanoparticles should be biocompatible while drug-loaded ones should display good therapeutic bioactivity. ${ }^{32,33}$ Therefore, the cytotoxicity of the DOX-free nanoparticles was analyzed via 3-(4,5-dimethylthiazol2-yl)-2,5-diphenyltetrazolium bromide (MTT) assay by culturing them against A549 cells (a carcinomic human alveolar basal epithelial cell line). The results indicate that cells treated by MPB nanohybrids displayed a high cell viability $(\sim 85 \%)$ even after cell culture time of $48 \mathrm{~h}$ and at nanoparticle concentration up to $7.50 \mu \mathrm{g} \mathrm{mL} \mathrm{m}^{-1}$ (Fig. 5). These mean that the nanoparticles are biocompatible. It can be seen from Fig. 6 that MDPB showed 


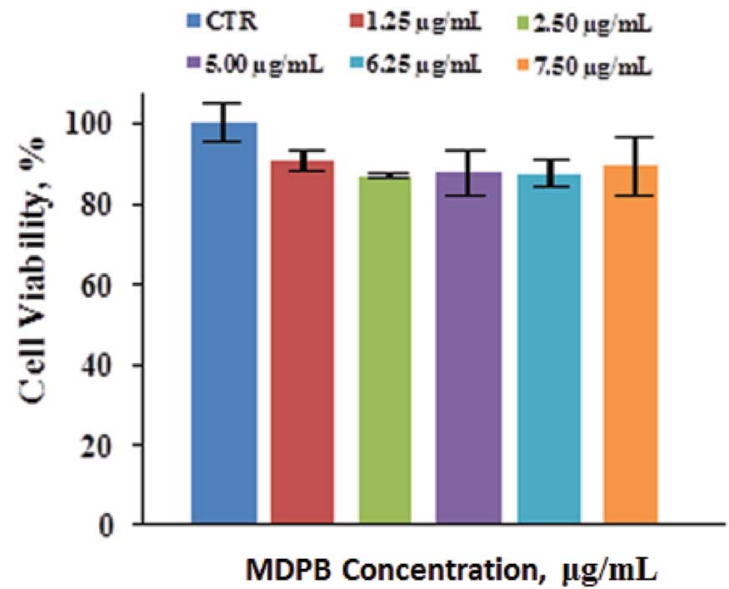

Fig. 5 Cell viability of A549 cells after $48 \mathrm{~h}$ incubation with MPB nanohybrids ( \pm standard deviation, $n=3$ ).

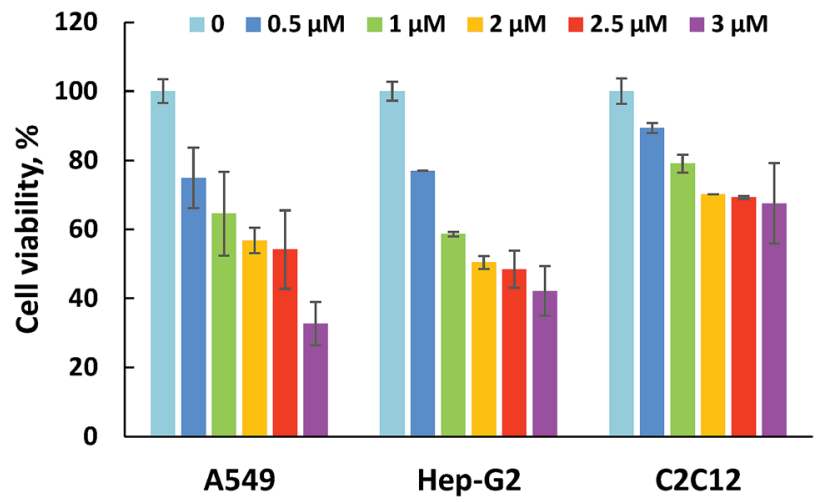

Fig. 6 Cell viability of A549 cells, Hep-G2 cells and C2C12 cells after $48 \mathrm{~h}$ incubation with MDPB_0.1 nanohybrids with different weight concentration of encapsulated DOX ( \pm standard deviation, $n=3$ ).

a dosage-dependent cytotoxicity towards A549 cells, Hep-G2 cells and C2C12 cells with IC50 values of 2.11, 2.03 and 6.90 $\mu \mathrm{M}$, revealing that the cytotoxic effect was only from the drug which was loaded within the nanohybrids and their specific cytotoxicity against cancer cells as compared to normal cells (Fig. 6). The high cytotoxicity may be associated with their charge-reversal ability, beneficial to an enhanced cell uptake capacity (Fig. 7). The biodegradability and good cytocompatibility of the MDPB nanoparticles, as well as their pH-sensitive drug release controllability make them promising for anticancer therapeutic delivery applications.

\section{Experimental}

\section{Materials and cells}

$\mathrm{N}$-Cetyltrimethylammonium bromide (CTAB), tetraethyl orthosilicate (TEOS) and triethylamine (TEA) were acquired from Shanghai Lingfeng Chemical Reagent Co. Ltd., China. Polyethylenimine (LPEI, $M_{\mathrm{w}}=1800 \mathrm{Da}$ ) with low molecular weight was bought from Aladdin Co. Ltd, China. Bovine serum albumin (BSA) was purchased from Shanghai Juyuan Co. Ltd,

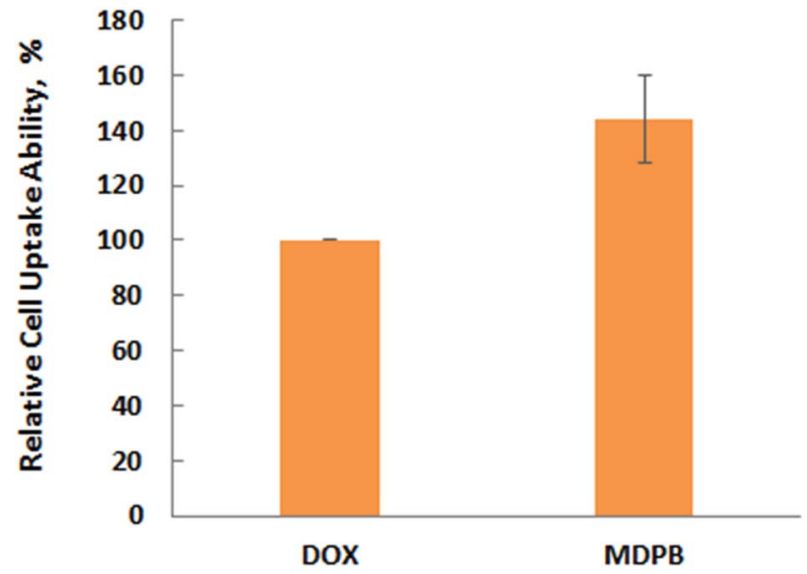

Fig. 7 Flow cytometry assay of A549 cells after $24 \mathrm{~h}$ incubation with free DOX and MDPB_0.1 nanohybrids with equivalent DOX concentration $(3.0 \mu \mathrm{m})$ ( \pm standard deviation, $n=3$ ).

China. Doxorubicin hydrochloride (DOX) was bought from Dalian MeiLun Biology Technology Co. Ltd, China. 3-(4,5Dimethyl-2-thiazolyl)-2,5-diphenyl-2- $H$-tetrazolium bromide (MTT) and 4',6-diamidino-2-phenylindole (DAPI) were received from Life Technology, USA. A549 cells (a carcinomic human alveolar basal epithelial cell line) and Hep-G2 cells (a human liver cancer cell line) were purchased from cell bank of Chinese Academy of Sciences, Shanghai, China. C2C12 cells (a mouse myoblast cell line) were purchased from American Type Culture Collection (ATCC, VA, USA).

\section{Preparation and characterization of MDPB nanoparticles}

Mesoporous silica nanoparticles (MSN) were prepared according to an emulsion method. ${ }^{18}$ Briefly, $1.5 \mathrm{~mL}$ TEOS was added in $20 \mathrm{~mL}$ aqueous solution containing $2.18 \mathrm{~g}$ CTAB and $0.08 \mathrm{~g}$ TEA under magnetic stirring at $95{ }^{\circ} \mathrm{C}$, and the reaction was maintained at the same conditions for another $4 \mathrm{~h}$. The precipitation was collected by centrifugation and 3 time water/ethanol wash, followed by 2 day reflux with the ethanol solution of hydrochloric acid $(10 \% \mathrm{v} / \mathrm{v})$ at $78{ }^{\circ} \mathrm{C}$. The mixture was filtered and lyophilized to obtain mesoporous silica nanoparticles (MSN).

For drug loading, $1 \mathrm{~mL}$ DOX aqueous solution $\left(2 \mathrm{mg} \mathrm{mL}^{-1}\right)$ was mixed with $19 \mathrm{~mL}$ phosphate-buffered saline (PBS, $\mathrm{pH}=$ 7.4) containing $10 \mathrm{mg}$ MSN under $400 \mathrm{rpm}$ stirring for $24 \mathrm{~h}$, followed by centrifugation/water wash $(10000 \mathrm{rpm}, 10 \mathrm{~min})$ thrice. The obtained DOX-loaded MSN were abbreviated as "MD". For surface modification, $10 \mathrm{mg}$ MD samples in $9 \mathrm{~mL}$ distilled water was mixed with $1 \mathrm{~mL}$ aqueous solution of LPEI $\left(0.2 \mathrm{mg} \mathrm{mL}^{-1}\right)$ under magnetic stirring for $1 \mathrm{~h}$. The mixture was then centrifuged/washed thrice to get MDP samples, which were resuspended into $9 \mathrm{~mL}$ water and treated with $1 \mathrm{~mL}$ aqueous solution containing different amount of BSA under stirring at room temperature for $12 \mathrm{~h}$. The solution then underwent centrifugation/wash to obtain MDPB nanoparticles. The unloaded DOX in the supernatant was evaluated by DOX fluorescence analysis $\left(\lambda_{\mathrm{ex}}=480 \mathrm{~nm}, \lambda_{\mathrm{em}}=580 \mathrm{~nm}\right)$ using 
a microplate reader (SpectraMax M2, Molecular Devices, USA) to calculate drug loading amount.

The Zeta potential of the nanoparticles at different $\mathrm{pH}$ values in PBS were analysed by a Zetasizer Instrument (Nano ZS, Malvern Instruments, UK) via a Dynamic Light Scattering (DLS) technique. The morphology of the nanoparticles was examined by transmission electron microscope (TEM, JEOL JEM-2100, Nikon, Japan) with an accelerating voltage of $120 \mathrm{kV}$. Before measurement, the samples were dispersed in ethanol $\left(0.5 \mathrm{mg} \mathrm{mL}{ }^{-1}\right)$ under sonication. The aqueous suspensions of the samples were dropped onto a 400 mesh copper grid, followed by air-drying before analysis.

\section{In vitro drug release study}

For the drug release experiments, DOX-loaded nanoparticles in $1 \mathrm{~mL}$ water containing equivalent DOX amount $(100 \mu \mathrm{g})$ was introduced in a dialysis membrane (MWCO: $14000 \mathrm{Da}$, Shanghai Yuan Ju Biological Technology Co. Ltd., Shanghai, China). Dialysis was then performed against $9 \mathrm{~mL}$ PBS solution, under different $\mathrm{pH}$ values $(7.4,6.5$ or 5.0$)$, at a temperature $37^{\circ} \mathrm{C}$. At different time intervals, an aliquot of the PBS solution $(100 \mu \mathrm{L})$ was taken out for spectrophotometrical analysis and refreshed with $100 \mu \mathrm{L}$ PBS solution. The released DOX was quantified by measuring the DOX fluorescence $\left(\lambda_{\mathrm{ex}}=480 \mathrm{~nm}\right.$, $\lambda_{\mathrm{em}}=580 \mathrm{~nm}$ ) using a microplate reader (SpectraMax M2, Molecular Devices, USA). The cumulative release $\left(C_{\mathrm{r}}\right)$ of DOX against time was obtained according to the equation:

$$
C_{\mathrm{r}}=100 \times W_{\mathrm{t}} / W_{\text {tot }}
$$

where $W_{\mathrm{t}}$ and $W_{\text {tot }}$ are the cumulative amount of drug released at time $t$, and the total drug contained in the nanohybrids used for drug release, respectively.

\section{Evaluation of cell viability}

A549 cancer cells (a carcinomic human alveolar basal epithelial cell line), Hep-G2 cancer cells (a human liver cancer cell line), together with $\mathrm{C} 2 \mathrm{C} 12$ normal cells (mouse myoblast cell line) as normal cell control, were incubated in flasks containing Dulbecco's modified Eagle medium (DMEM) and 10\% fetal bovine serum (FBS) in a humidified atmosphere and $5 \%$ of carbon dioxide in a Corning culturist (incubator) at $37^{\circ} \mathrm{C}$.

The cytotoxicity of DOX-free or loaded nanoparticles was evaluated by examining the viability of A549 cells using a MTT assay. Briefly, cells were incubated in 96-well plate at a density of 5000 cells per well. After 1 day, the cultured DMEM solution was replaced with $200 \mu \mathrm{L}$ fresh DMEM solutions of DOX-free and DOX-loaded nanoparticles. Subsequently, cells were incubated for $48 \mathrm{~h}$ at $37{ }^{\circ} \mathrm{C}$ before the MTT assay. For MTT assay, a $30 \mu \mathrm{L}$ MTT solution was added to each well. After further incubation for $4 \mathrm{~h}$ at $37^{\circ} \mathrm{C}, 200 \mu \mathrm{L}$ DMSO was added to each well to replace the culture medium and dissolve the insoluble formazan crystals. The absorbance at $492 \mathrm{~nm}$ was measured by using the UV spectrophotometer. The relative cell viability was demonstrated as $\mathrm{OD}_{\text {test }} / \mathrm{OD}_{\text {control }} \times 100 \%$.
For cell uptake quantification, intracellular drug accumulation was investigated via flow cytometry assay. Briefly, A549 cells (10 000 cells per well in a 6-well plate) were incubated against free DOX, MDPB_0.1 in DMEM containing $3.0 \mu \mathrm{M}$ equivalent DOX. After $24 \mathrm{~h}$ incubation, cells were washed with PBS, trypsinized and recollected, which were re-suspended in PBS $(0.5 \mathrm{~mL})$ for a flow cytometry assay.

\section{Conclusions}

In summary, we develop an effective approach to fabricate a kind of stimulative mesoporous silica nanoparticles. An anticancer drug, doxorubicin (DOX), was firstly loaded into mesoporous silica nanoparticles (MSN), which were coated with cationic oligomer (LPEI) as well as natural negative protein (BSA) onto their surface. The variation of LPEI and BSA contents allowed for controllable adjustment of their surface charge to acquire charge-reversal ability. The resulting nanoparticles (MDPB) presented $\mathrm{pH}$ sensitivity in DOX delivery under conditions mimicking intracellular conditions and acidic tumor microenvironments, resulting in good cytotoxicity against cancer cells.

\section{Conflicts of interest}

There are no conflicts of interest to declare.

\section{Acknowledgements}

The Public Projects of Zhejiang Province (2015C31040) and Project of Zhejiang Province Department of Education (Y201328505) were acknowledged. The authors appreciated the support of the Shanghai Municipal Natural Science Foundation (15ZR1408500) and Shanghai International Cooperation Program (15520721200).

\section{Notes and references}

1 Y. Barenholz, J. Controlled Release, 2012, 160, 117-134.

2 Y. Li, Y. Xiao and C. Liu, Chem. Rev., 2017, 117, 4376-4421.

3 E. Miele, G. P. Spinelli, E. Miele, F. Tomao and S. Tomao, Int. J. Nanomed., 2009, 4, 99-105.

4 Y. Li and C. Liu, Nanoscale, 2017, 9, 4862-4874.

5 S. D. Huo, H. L. Ma, K. Y. Huang, J. Liu, T. Wei, S. B. Jin, J. C. Zhang, S. T. He and X. J. Liang, Cancer Res., 2013, 73, 319-330.

6 Y. L. Li, D. Maciel, J. Rodrigues, X. Y. Shi and H. Tomas, Chem. Rev., 2015, 115, 8564-8608.

7 E. C. Cho, J. W. Xie, P. A. Wurm and Y. N. Xia, Nano Lett., 2009, 9, 1080-1084.

8 S. E. A. Gratton, P. A. Ropp, P. D. Pohlhaus, J. C. Luft, V. J. Madden, M. E. Napier and J. M. DeSimone, Proc. Natl. Acad. Sci. U. S. A., 2008, 105, 11613-11618.

9 D. Oupicky, M. Ogris, K. A. Howard, P. R. Dash, K. Ulbrich and L. W. Seymour, Mol. Ther., 2002, 5, 463-472. 
10 K. Xiao, Y. P. Li, J. T. Luo, J. S. Lee, W. W. Xiao, A. M. Gonik, R. G. Agarwal and K. S. Lam, Biomaterials, 2011, 32, 34353446.

11 M. G. V. Heiden, L. C. Cantley and C. B. Thompson, Science, 2009, 324, 1029-1033.

12 J. Y. Han and K. F. Burgess, Chem. Rev., 2010, 110, 27092728.

13 P. Zhang, T. Wu and J. L. Kong, ACS Appl. Mater. Interfaces, 2014, 6, 17446-17453.

14 M. Benezra, O. Penate-Medina, P. B. Zanzonico, D. Schaer, H. Ow, A. Burns, E. DeStanchina, V. Longo, E. Herz, S. Iyer, J. Wolchok, S. M. Larson, U. Wiesner and M. S. Bradbury, J. Clin. Invest., 2011, 121, 2768-2780.

15 M. Q. Wang, X. Li, Y. J. Ma and H. C. Gu, Int. J. Pharm., 2013, 448, 51-57.

16 I. Y. Park, I. Y. Kim, M. K. Yoo, Y. J. Choi, M. H. Cho and C. S. Cho, Int. J. Pharm., 2008, 359, 280-287.

17 B. Trzebicka, R. Szweda, D. Kosowski, D. Szweda and L. Otulakowski, Prog. Polym. Sci., 2017, 68, 35-76.

18 Y. Wang, Y. Sun, J. Wang, Y. Yang, Y. Li, Y. Yuan and C. Liu, ACS Appl. Mater. Interfaces, 2016, 8, 17166-17175.

19 Q. Zhao, J. Liu, W. Zhu, C. Sun, D. Di, Y. Zhang, P. Wang, Z. Wang and S. Wang, Acta Biomater., 2015, 23, 147-156.

20 X. Chen and Z. Liu, J. Mater. Chem. B, 2016, 4, 4382-4388.

21 X. Du, L. Xiong, S. Dai and S. Z. Qiao, Adv. Healthcare Mater., 2015, 4, 771-781.
22 A. Nouri, R. Castro, V. Kairys, J. L. Santos, J. Rodrigues, Y. L. Li and H. Tomas, J. Mater. Sci.: Mater. Med., 2012, 23, 2967-2980.

23 V. M. Platt and F. C. Szoka, Mol. Pharm., 2008, 5, 474-486.

24 M. Goncalves, P. Figueira, D. Maciel, J. Rodrigues, X. Qu, C. S. Liu, H. Tomas and Y. L. Li, Acta Biomater., 2014, 10, 300-307.

25 G. Y. Wang, D. Maciel, Y. L. Wu, J. Rodrigues, X. Y. Shi, Y. Yuan, C. S. Liu, H. Tomas and Y. L. Li, ACS Appl. Mater. Interfaces, 2014, 6, 16687-16695.

26 M. Oishi, S. Sumitani and Y. Nagasaki, Bioconjugate Chem., 2007, 18, 1379-1382.

27 J. Yang, H. T. Chen, I. R. Vlahov, J. X. Cheng and P. S. Low, J. Pharmacol. Exp. Ther., 2007, 321, 462-468.

28 M. Goncalves, P. Figueira, D. Maciel, J. Rodrigues, X. Y. Shi, H. Tomas and Y. L. Li, Macromol. Biosci., 2014, 14, 110-120.

29 J. Y. Song, J. Cha, J. Lee and J. H. Roe, Eukaryotic Cell, 2006, 5, 1857-1865.

30 R. Cheng, F. Feng, F. H. Meng, C. Deng, J. Feijen and Z. Y. Zhong, J. Controlled Release, 2011, 152, 2-12.

31 Y. Li, D. Maciel, J. Rodrigues and X. Shi, Chem. Rev., 2015, 115, 8564-8608.

32 D. Maciel, P. Figueira, S. L. Xiao, D. M. Hu, X. Y. Shi, J. Rodrigues, H. Tomas and Y. L. Li, Biomacromolecules, 2013, 14, 3140-3146.

33 M. Goncalves, D. Maciel, D. Capelo, S. L. Xiao, W. J. Sun, X. Y. Shi, J. Rodrigues, H. Tomas and Y. L. Li, Biomacromolecules, 2014, 15, 492-499. 